\title{
ON CHAINS OF CENTERED VALUATIONS
}

\section{RACHID CHIBLOUN}

Received 5 June 2002

\begin{abstract}
We study chains of centered valuations of a domain $A$ and chains of centered valuations of $A\left[X_{1}, \ldots, X_{n}\right]$ corresponding to valuations of $A$. Finally, we make some applications to chains of valuations centered on the same ideal of $A\left[X_{1}, \ldots, X_{n}\right]$ and extending the same valuation of $A$.
\end{abstract}

2000 Mathematics Subject Classification: 13A18, 13F20.

1. Introduction and preliminary results. All rings will be commutative with unit element. The field of fractions of a domain $R$ will be denoted by $\operatorname{Fr}(R)$. Let $R \subseteq S$ be domains and take a prime ideal $p \in \operatorname{Spec}(R)$. Then we write $k(p)=$ $\operatorname{Fr}(R / p)$ and denote the transcendence degree of $\operatorname{Fr}(S)$ over $\operatorname{Fr}(R)$ by $\operatorname{tr} \operatorname{deg}_{R}^{S}$. We will use the following notation: $R[n]=R\left[X_{1}, \ldots, X_{n}\right], p[n]=p\left[X_{1}, \ldots, X_{n}\right]$, and $\operatorname{Fr}(R)(n)=\operatorname{Fr}(R)\left(X_{1}, \ldots, X_{n}\right)$. Let $P \in \operatorname{Spec}(R[n]), P$ lies over $p$ if $P \cap R=p$. We call $P \in \operatorname{Spec}(R[1])$ a superior of $p$ if $P$ lies over $p$ and $P \neq p$ [1]. Let $A$ be a subring of a field $K, L / K$ a field extension, and $v$ a valuation on $K$. The subring $A_{v}=\{x \in K \mid v(x) \geq 0\}$ is the valuation ring associated to $v, m(v)=\{x \in$ $K \mid v(x)>0\}$ is its maximal ideal, and $k_{v}=A_{v} / m(v)$ is its residue field. The valuation $v$ is positive on $A$ if $A \subseteq A_{v}$, and then $v$ is a valuation on $A$. The prime ideal $m(v) \cap A$ is called the center of $v$ on $A$. The valuation $v$ is called trivial if $A_{v}=K$. If $v^{\prime}$ is a valuation on $k_{v}$, then the set $\left\{x \in K \mid x \in A_{v}, \bar{x} \in A_{v^{\prime}}\right\}$ is a valuation ring on $K$. The valuation associated to this valuation ring is called the composite valuation and is denoted by $v_{1}=v^{\prime} v$.

Let $v, v^{\prime}$ be valuations on $K$. By definition, $v \leq v^{\prime}$ if one of the following equivalent conditions is satisfied:

(1) $A_{v^{\prime}} \subseteq A_{v}$,

(2) $m(v) \subseteq m\left(v^{\prime}\right)$,

(3) $v^{\prime}=v^{\prime \prime} v$ for some valuation on $k_{v}$;

$v$ and $v^{\prime}$ are called equivalent if $A_{v^{\prime}}=A_{v} ; v<v^{\prime}$ if $v \leq v^{\prime}$ but not equivalent. A valuation $w$ on $L$ is an extension of $v$ if $A_{v}=A_{w} \cap K$. The valuation $w$ on $K(X)$ given by

$$
w\left(\sum_{i=0}^{n} a_{i}(X-a)^{i}\right)=\inf \left\{v\left(a_{i}\right) \mid 0 \leq i \leq n\right\}
$$

is called the canonical extension of $v$ to $K(X)$. We have that $k_{w}=k_{v}(X)$. 
The following classical results will be used in this paper; the proofs can be found in [2, Proposition 1.2], [4, Theorem 1.5], and [6, Propositions 1.1, 1.3, and 1.4].

Proposition 1.1. Let $v$ be a valuation on $K$ and $w_{0}<w_{1}$ two valuations on $L$ extending $v$. If $\operatorname{tr} \operatorname{deg}_{k_{v}}^{k_{w}}$ is finite, then

$$
\operatorname{tr} \operatorname{deg}_{k_{v}}^{k_{w_{1}}}<\operatorname{trdeg} k_{k_{v}}^{k_{w_{0}}} .
$$

Proposition 1.2. Let $v_{0}<v_{1}$ be two valuations on $K$ and $w_{1}$ a valuation on $L$ extending $v_{1}$. Then there exists a valuation $w_{0}$ on $L$ extending $v_{0}$, with $w_{0}<w_{1}$.

Proposition 1.3. Let $w$ be a valuation on $L$ and $v$ its restriction to $K$. If $\operatorname{tr} \operatorname{deg}_{K}^{L}$ is finite, then

$$
\operatorname{tr} \operatorname{deg}_{k_{v}}^{k_{w}} \leq \operatorname{tr} \operatorname{deg}_{K}^{L}
$$

Proposition 1.4. If $p \subseteq q$ in $\operatorname{Spec}(A)$ and if $v_{0}$ is a valuation of $K$ with center $p$ on $A$, then there exists a valuation $v_{1}$ of $K$ with center $q$ on $A$ such that $v_{0} \leq v_{1}$.

THEOREM 1.5. Let $f: A \rightarrow B$ be a homomorphism of domains. Then there exist an algebraic extension $L^{\prime}$ of $\operatorname{Fr}(B)$ and a valuation $v$ on $K$ with center $\operatorname{Ker}(f)$ on A such that

$$
A / \operatorname{Ker}(f) \subseteq k_{v} \subseteq L
$$

In this paper, we will study chains of valuations of a polynomial ring $A\left[X_{1}\right.$, $\left.\ldots, X_{n}\right]$ and of a field extension $F$ of $\operatorname{Fr}(A)$. We give the length of chains of valuations which pass through a given valuation, and we characterize when a valuation is maximal or minimal in the following situations:

(a) all the valuations are centered on the same ideal,

(b) all the valuations extend the same valuation of $\operatorname{Fr}(A)$.

Then we study chains of centered valuations on a domain $A$ and chains of centered valuations on $A\left[X_{1}, \ldots, X_{n}\right]$ corresponding to valuations on $A$. Finally, we give some applications to chains of valuations centered on the same ideal of $A\left[X_{1}, \ldots, X_{n}\right]$ and extending the same valuation on $A$.

2. Valuations centered on the same ideal. Throughout this section, $K$ is the quotient field of an integral domain $A, L$ is a field extension of $K$, and $v$ is a valuation on $A$.

Proposition 2.1. There exist $n+1$ valuations $w_{0}<\cdots<w_{n}$ on $A[n]$ extending $v$ in such a way that, for each $i \in\{0, \ldots, n\}$,

$$
\operatorname{tr} \operatorname{deg}_{k_{v}}^{k_{w_{i}}}=n-i
$$


Proof. Let $k=k_{v}$ and let $w_{0}$ be the canonical extension of $v$ to $K(X)$. It is well known that $k_{w_{0}}=k(X)$. Let $w$ be a valuation on $k(X)$, positive on $k[X]$ and with center $(X)$ on $k[X]$, and $w_{1}=w w_{0}$ the composite valuation of $w$ and $w_{0}$. The valuation $w_{0}<w_{1}$ as $w$ is not trivial, so $A_{w_{1}} \cap K \subseteq A_{w_{0}} \cap K=A_{v}$ and it is easy to see that

$$
A_{v} \subseteq\left\{z \in K(X) \mid z \in A_{w_{0}}, \bar{z} \in A_{w}\right\} \cap K
$$

Therefore, $w_{1}$ extends $v$. As $A[X] \subseteq A_{w_{0}}$ and $k[X] \subseteq A_{w}, X \in A_{w_{0}}$ and $\bar{X}=X \in$ $A_{w}$, that is, $X \in A_{w_{1}}$, and $w_{1}$ is a valuation of $A[X]$. We have $\operatorname{tr} \operatorname{deg}_{k_{v}}^{k_{w_{0}}}=1$, and according to Proposition 1.1, $0 \leq \operatorname{tr} \operatorname{deg}_{k_{v}}^{k_{w_{1}}}<\operatorname{tr} \operatorname{deg}_{k_{v}}^{k_{w_{0}}}=1$, so $\operatorname{tr} \operatorname{deg}_{k_{v}}^{k_{w_{1}}}=0$.

Let $n>1$ and suppose that the property is true for $n-1$. There exists $v_{0}<$ $v_{1}$, two valuations of $A\left[X_{1}\right]$ extending $v$, and for each $i \in\{0,1\}, \operatorname{tr} \operatorname{deg}_{k_{v}}^{k_{v_{i}}}=1-$ $i$. There exists $n$ valuations $w_{1}<\cdots<w_{n}$ of $A[n]$ extending $v_{1}$, and for each $i \in\{1, \ldots, n\}$, we have that $\operatorname{tr}^{\operatorname{deg}_{k_{v_{1}}}}=n-i$. According to Proposition 1.2, there exists a valuation $w_{0}^{\prime}$ of $K\left(X_{1}, \ldots, X_{n}\right)$ extending $v_{0}$ and $w_{0}^{\prime}<w_{1}<\cdots<w_{n}$. The valuation $w_{0}^{\prime}$ is a valuation of $A[n]$ because $A[n] \subseteq A_{w_{1}} \subset A_{w_{0}^{\prime}}$. For each $i \in\{1, \ldots, n\}, w_{i}$ extends $v$ and $\operatorname{trdeg}_{k_{v}}^{k_{w_{i}}}=\operatorname{trdeg}_{k_{v_{1}}}^{k_{w_{i}}}+\operatorname{tr} \operatorname{deg}_{k_{v}}^{k_{v_{1}}}=n-i, w_{0}^{\prime}$ extends $v$, and

$$
\begin{aligned}
\operatorname{trdeg}_{k_{v}}^{k_{w_{0}^{\prime}}} & =\operatorname{trdeg}{ }_{k_{v_{0}}}^{{ }_{w_{0}^{\prime}}}+\operatorname{trdeg}_{k_{v}}^{k_{v_{0}}} \\
& =\operatorname{trdeg}{ }_{k_{v_{0}}}^{k_{w_{0}^{\prime}}}+1>\operatorname{tr} \operatorname{deg}_{k_{v}}^{k_{w_{1}}}=n-1,
\end{aligned}
$$

according to Proposition $1.3, n-1<\operatorname{tr} \operatorname{deg}_{k_{v}}^{k_{w_{0}^{\prime}}} \leq n$, that is, $\operatorname{trdeg}_{k_{v}}^{k_{w_{0}^{\prime}}}=n$.

COROLLARY 2.2. If $\operatorname{tr} \operatorname{deg}_{K}^{L}=n$, then there exist $n+1$ valuations $w_{0}<\cdots<$ $w_{n}$ on $L$ extending $v$ such that $\operatorname{tr} \operatorname{deg}_{k_{v}}^{k_{w_{i}}}=n-i$ for all $i \in\{0, \ldots, n\}$.

Proof. Let $\left\{x_{1}, \ldots, x_{n}\right\}$ be a transcendence basis of $L$ over $K, v$ a valuation of $A_{v}$, and $A_{v}\left[x_{1}, \ldots, x_{n}\right] \cong A_{v}\left[X_{1}, \ldots, X_{n}\right]$. According to Proposition 2.1, there exist $n+1$ valuations $v_{0}<\cdots<v_{n}$ on $K\left(x_{1}, \ldots, x_{n}\right)$ extending $v$ such that $\operatorname{tr} \operatorname{deg}_{k_{v}}^{k_{v_{i}}}=n-i$ for each $i \in\{0, \ldots, n\}$. Let $w_{n}$ be a valuation of $L$ extending $v_{n}$. Applying Proposition 1.2, we obtain $n+1$ valuations $w_{0}<\cdots<w_{n}$ of $L$ such that for each $i \in\{0, \ldots, n\}, w_{i}$ prolongs $v_{i}$, then $w_{i}$ prolongs $v$, and

$$
\operatorname{tr} \operatorname{deg}_{k_{v}}^{k_{w_{i}}}=\operatorname{tr} \operatorname{deg}_{k_{v_{i}}}^{k_{w_{i}}}+\operatorname{tr} \operatorname{deg}_{k_{v}}^{k_{v_{i}}}=n-i
$$

LEMMA 2.3. Let $v_{0}$ be a valuation on $L$ with center $q$ on $A$. For each $k \in \mathbb{N}$ strictly smaller than $\operatorname{tr} \operatorname{deg}_{k(q)}^{k_{v_{0}}}$, there exists a valuation $v_{1}$ of $L$ with center $q$ on A such that $v_{0}<v_{1}$ and $\operatorname{tr} \operatorname{deg}_{k(q)}^{k v_{1}}=k$. 
Proof. Let $\left\{z_{1}, \ldots, z_{k+1}\right\}$ be a family of elements of $k_{v_{0}}$, algebraically independent over $k(q)$. According to Theorem 1.5, there exist an algebraic extension $L^{\prime}$ of $k(q)\left(z_{1}, \ldots, z_{k}\right)$ and a valuation $v^{\prime}$ of $k_{v_{0}}$ with center $\left(z_{k+1}\right)$ on $(A / q)\left[z_{1}, \ldots, z_{k+1}\right]$, such that $(A / q)\left[z_{1}, \ldots, z_{k}\right] \subseteq k_{v^{\prime}} \subseteq L^{\prime}$. Let $v_{1}=v^{\prime} v_{0}$ be the composite valuation of $v^{\prime}$ with $v_{0}, v_{1}$ is a valuation of $L$. For each $b \in A, b \in A_{v_{0}}$ and $\bar{b} \in A / q \subseteq A_{v^{\prime}}$, that is, $b \in A_{v_{1}}$, and if $a \in m\left(v_{1}\right) \cap A$, then $\bar{a} \in\left(m\left(v_{1}\right) / m\left(v_{0}\right)\right) \cap(A / q)=m\left(v^{\prime}\right) \cap(A / q)=(0)$, that is, $a \in q$ and $m\left(v_{1}\right) \cap A \subseteq q$ or $q=m\left(v_{0}\right) \cap A \subseteq m\left(v_{1}\right) \cap A$; therefore the center of $v_{1}$ on $A$ is $q$. As $A_{v^{\prime}}=A_{v_{1}} / m\left(v_{0}\right), m\left(v^{\prime}\right)=m\left(v_{1}\right) / m\left(v_{0}\right)$ and $k_{v^{\prime}}=A_{v^{\prime}} / m\left(v^{\prime}\right)=$ $A_{v_{1}} / m\left(v_{1}\right)$. Thus, $v_{0}<v_{1}$ and $\operatorname{trdeg}_{k(q)}^{k_{v_{1}}}=\operatorname{trdeg}_{k(q)}^{k_{v^{\prime}}}=\operatorname{trdeg}_{k(q)}^{k(q)\left(z_{1}, \ldots, z_{k}\right)}=k$.

THEOREM 2.4. Let $w$ be a valuation on $L$ with center $q$ on $A$. Then $\operatorname{tr} \operatorname{deg}_{k(q)}^{k_{w}}$ is the supremum of all natural numbers $\bar{k}$ for which there exists a chain of valuations $w=w_{0}<\cdots<w_{\bar{k}}$ on $L$, with center $q$ on $A$.

Proof. Suppose that we have a chain of valuations $w=w_{0}<\cdots<w_{k}$ on $L$, with center $q$ on $A$. If $\operatorname{tr}_{\operatorname{deg}_{k(q)}^{k_{w}}}$ is finite, then it follows from Proposition 1.1 that

$$
0 \leq \operatorname{tr} \operatorname{deg}_{k(q)}^{k_{w_{k}}}<\cdots<\operatorname{trdeg}_{k(q)}^{k_{w_{0}}}=\operatorname{trdeg}_{k(q)}^{k_{w}},
$$

and consequently $k \leq \operatorname{tr} \operatorname{deg}_{k(q)}^{k_{w}}$. This proves that $\bar{k} \leq \operatorname{tr} \operatorname{deg}_{k(q)}^{k_{w}}$.

To prove the converse inequality, we consider two different cases:

(a) $\operatorname{tr} \operatorname{deg}_{k(q)}^{k_{w}}=k_{1} \in \mathbb{N}$ is finite. If $k_{1}=0$, then there is nothing to prove. Take $k_{1}>0$. By Lemma 2.3, there exists a valuation $w_{1}$ on $L$ with center $q$ on $A$ such that $w<w_{1}$ and $\operatorname{tr}_{\operatorname{deg}^{\prime}(q)}^{k_{w_{1}}}=k_{1}-1$. Using an easy induction argument, we find $k_{1}+1$ valuations with $w=w_{0}<\cdots<w_{k}$ on $L$, all with center $q$ on $A$;

(b) $\operatorname{tr} \operatorname{deg}_{k(q)}^{k w}=\infty$. By Lemma 2.3, we can find, for every $k \in \mathbb{N}$, a valuation $w_{1}$ on $L$ with center $q$ on $A$ such that $\operatorname{tr} \operatorname{deg}_{k(q)}^{k_{w_{1}}}=k$. It then follows from (a) that there exists a chain of valuations $w=w_{0}<\cdots<w_{k}$ of $L$, all with center $q$ on $A$. We can do this for every $k \in \mathbb{N}$, hence the supremum is infinite.

LEMMA 2.5. Let $w$ be a valuation on $A[n]$ with center $q$ on $A$.

(a) If $\operatorname{tr} \operatorname{deg}_{k(q)}^{k w}=\infty$, then for every $k \in \mathbb{N}$, there exists a valuation $w_{1}$ on $A[n]$ with center $q$ on $A$ such that $w<w_{1}$ and $\operatorname{tr}_{\operatorname{deg}^{\prime}(q)}^{k_{w_{1}}}=k$.

(b) If $\operatorname{tr} \operatorname{deg}_{k(q)}^{k_{w}}=k \in \mathbb{N}$, then there exists a chain of valuations $w=w_{0}<$ $\cdots<w_{k}$ on $A[n]$, all with center $q$ on $A$.

Proof. (a) Let $Q$ be the center of $w$ on $A[n]$ and $k_{1}=\operatorname{trdeg}_{A / q}^{A[n] / Q}$. We know that $k_{1}=n-\operatorname{ht}(Q / q[n])$, where $\mathrm{ht}(Q / q[n])$ means the height of the prime ideal $Q / q[n]$, and there exists a chain $Q=Q_{0} \subset \cdots \subset Q_{k_{1}}$ of prime ideals of $A[n]$, all lying over $q$. 
Assume first that $k<k_{1}$; then there exists $i \in\left\{1, \ldots, k_{1}\right\}$ such that $\operatorname{tr} \operatorname{deg}_{A / q}^{A[n] / Q_{i}}$ $=k$. Let $w^{\prime \prime}$ be a valuation on $A[n]$ with center $Q_{i}$ and $w<w^{\prime \prime}$ (see Proposition 1.4). According to Lemma 2.3, there exists a valuation $w_{1}$ on $A[n]$ with center $Q_{i}$ such that $w^{\prime \prime} \leq w_{1}$ and $\operatorname{tr} \operatorname{deg}_{A[n] / Q_{i}}^{k_{w_{1}}}=0$. Thus, $w<w_{1}$ and $\operatorname{tr} \operatorname{deg}_{k(q)}^{k_{w_{1}}}=$ $\operatorname{tr} \operatorname{deg}_{A[n] / Q_{i}}^{k_{w_{1}}}+\operatorname{tr} \operatorname{deg}_{A / q}^{A[n] / Q_{i}}=k$.

Now assume that $k \geq k_{1}$ and let $\alpha=k-k_{1}$. By Theorem 2.4, there exists a valuation $w_{1}$ on $A[n]$ with center $Q$ such that $w<w_{1}$ and $\operatorname{tr} \operatorname{deg}_{A[n] / Q}^{k_{w_{1}}}=\alpha$, hence

$$
\operatorname{tr} \operatorname{deg}_{A / q}^{k_{w_{1}}}=\operatorname{trdeg}_{A[n] / Q}^{k_{w_{1}}}+\operatorname{tr} \operatorname{deg}_{A / q}^{A[n] / Q}=\alpha+k_{1}=k
$$

(b) Let $Q$ be the center of $w$ on $A[n]$ and $k_{1}=\operatorname{trdeg}_{A[n] / Q}^{k_{w}}$. According to Theorem 2.4, there exists a chain of valuations $w=w_{0}<\cdots<w_{k_{1}}$ on $A[n]$, all with center $Q$, such that $\operatorname{tr} \operatorname{deg}_{A[n] / Q}^{k_{w_{i}}}=k_{1}-i$ for each $i \in\left\{0, \ldots, k_{1}\right\}$. Let $\alpha=\operatorname{tr} \operatorname{deg}_{A / q}^{A[n] / Q}$; then there exists a chain $Q=Q_{0} \subset \cdots \subset Q_{\alpha}$ of prime ideals of $A[n]$ lying over $q$. According to Proposition 1.4 , there exist $\alpha+1$ valuations $w_{k_{1}}<\cdots<w_{k_{1}+\alpha}=w_{k}$ on $A[n]$ such that $w_{k_{1}+j}$ has center $Q_{j}$ on $A[n]$ for each $j \in\{0, \ldots, \alpha\}$. Therefore, $w_{k_{1}+j}$ has center $q$ on $A$, and the chain of valuations $w_{0}<\cdots<w_{k}$ meets the requirements.

THEOREM 2.6. Let $w$ be a valuation on $A[n]$ with center $q$ on $A$. Then $\operatorname{tr} \operatorname{deg}_{A / q}^{k_{w}}$ is the supremum of all natural numbers $\bar{k}$ such that there exists a chain of valuations $w=w_{0}<\cdots<w_{\bar{k}}$ on $A[n]$ with center $q$ on $A$.

Proof. Let $w=w_{0}<\cdots<w_{k}$ be a chain of valuations on $A[n]$ with center $q$ on $A$. If $\operatorname{tr} \operatorname{deg}_{A / q}^{k_{w}}$ is finite, then $0 \leq \operatorname{tr} \operatorname{deg}_{A / q}^{k_{w_{k}}}<\cdots<\operatorname{tr} \operatorname{deg}_{A / q}^{k_{w_{0}}}$, so $k \leq \operatorname{tr} \operatorname{deg}_{A / q}^{k_{w}}$, and it follows that $\bar{k}<\operatorname{tr} \operatorname{deg}_{A / q}^{k w}$.

Take $k \leq \operatorname{tr} \operatorname{deg}_{A / q}^{k_{w}}$. We distinguish two cases:

(1) $\operatorname{tr} \operatorname{deg}_{A / q}^{k_{w}}$ is finite. It follows from Lemma 2.5(b) that there exists a chain of valuations $w=w_{0}<\cdots<w_{k}$ on $A[n]$ with center $q$ on $A$;

(2) $\operatorname{tr} \operatorname{deg}_{A / q}^{k_{w}}$ is infinite. It follows from Lemma 2.5(a) that there exists a valuation $w_{1}$ on $A[n]$ with center $q$ on $A$ such that $w<w_{1}$ and $\operatorname{tr} \operatorname{deg}_{A / q}^{k w_{1}}=k$. In both cases, we obtain the existence of a chain of valuations $w=w_{0}<\cdots<$ $w_{k}$ on $A[n]$, all with center $q$ on $A$.

Proposition 2.7. Let $w$ be a valuation on $A[n]$ (resp., $L$ ) extending $v$. Then $\operatorname{tr} \operatorname{deg}_{k_{v}}^{k_{w}}$ is the supremum of the set of integers $k$ such that there exists a chain of valuations $w=w_{0}<\cdots<w_{k}$ on $A[n]$ (resp., L) extending $v$.

Proof. Let $w^{\prime}$ be a valuation on $K(n)$ (resp., $L$ ). We first show that $w^{\prime}$ is a valuation on $A[n]$ (resp., $L$ ) extending $v$ if and only if $w^{\prime}$ is a valuation on $A_{v}[n]$ (resp., $L$ ) with center $m(v)$ on $A_{v}$.

First, assume that $w^{\prime}$ is a valuation on $A[n]$ (resp., $L$ ) extending $v$. Then $A_{w^{\prime}} \cap K=A_{v}$ and $w^{\prime}$ is a valuation on $A[n]$ (resp., $L$ ), hence $w^{\prime}$ is a valuation 
on $A_{v}[n]$ (resp., $L$ ) and

$$
m\left(w^{\prime}\right) \cap A_{v}=m\left(w^{\prime}\right) \cap K \cap A_{v}=m(v) \cap A_{v}=m(v) .
$$

Conversely, $A[n] \subseteq A_{v}[n] \subseteq A_{w^{\prime}}$, therefore $A_{v} \subseteq A_{w^{\prime}} \cap K$. If $z \in A_{w^{\prime}} \cap K$ and $z \notin A_{v}$, then $z^{-1} \in m(v)=m\left(w^{\prime}\right) \cap K$, a contradiction. Hence, $A_{w^{\prime}} \cap K=A_{v}$ and $w^{\prime}$ extends $v$.

To finish the proof, it suffices to apply Theorems 2.4 and 2.6 to $w$ and $m(v)$ $\in \operatorname{Spec}\left(A_{v}\right)$.

COROLLARY 2.8. (a) Let $w$ be a valuation on $A[n]$ (resp., $L$ ) with center $q$ on $A$. Then $\operatorname{ht}\left(m(w) / q A_{w}\right)$ is the supremum $\bar{k}$ of the integers $k$ for which there exists a chain of valuations $w_{k}<\cdots<w_{0}=w$ on $A[n]$ (resp., $L$ ) with center $q$ on $A$.

(b) Let $w$ be a valuation on $A[n]$ (resp., L) extending $v$. Then ht $(m(w) /$ $\left.m(v) A_{w}\right)$ is the supremum $\bar{k}$ of the integers $k$ for which there exists a chain of valuations $w_{k}<\cdots<w_{0}=w$ on $A[n]$ (resp., L) extending $v$.

Proof. (a) Let $w_{k}<\cdots<w_{0}=w$ be a chain of valuations on $A[n]$ (resp., $L$ ) with center $q$ on $A$. We have $q A_{w} \subseteq m\left(w_{k}\right) \subset \cdots \subset m\left(w_{0}\right)=m(w)$, and therefore $\mathrm{ht}\left(m(w) / q A_{w}\right) \geq \bar{k}$.

Conversely, let $k_{1} \in \mathbb{N}$ with $k_{1} \leq \mathrm{ht}\left(m(w) / q A_{w}\right)$. Then there exists a chain $P_{k_{1}} \subset \cdots \subset P_{0}=m(w)$ in $\operatorname{Spec}\left(A_{w}\right)$ such that $P_{k_{1}} \cap A=q$. The valuation rings $A_{w}=\left(A_{w}\right)_{P_{0}} \subset \cdots \subset\left(A_{w}\right)_{P_{k_{1}}}$ of $K(n)$ (resp., $L$ ) are all with center $q$ on $A$. For each $i \in\left\{0, \ldots, k_{1}\right\}$, let $w_{i}$ be the valuation on $K(n)$ (resp., $L$ ) associated to $\left(A_{w}\right)_{P_{i}}$. Then $w_{k_{1}}<\cdots<w_{0}=w$ are valuations on $A(n)$ (resp., $L$ ), all with center $q$ on $A$.

(b) The proof follows immediately from (a) and the proof of Proposition 2.7.

COROLlary 2.9. (a) A valuation $w$ is a maximal (resp., minimal) element in the set of valuations on $A[n]$ (resp., L) extending $v$ if and only if $\operatorname{tr} \operatorname{deg}_{k_{v}}^{k_{w}}=0$ (resp., ht $\left.\left(m(w) / m(v) A_{w}\right)=0\right)$.

(b) A valuation $w$ is a maximal (resp., minimal) element in the set of valuations on $A[n]$ (resp., $L$ ) with center $q$ on $A$ if and only if $\operatorname{tr} \operatorname{deg}_{A / q}^{k_{w}}=0$ (resp., $\left.\mathrm{ht}\left(m(w) / q A_{w}\right)=0\right)$.

COROLLARY 2.10. Let $w$ be a valuation on $A[n]$ (resp., $L$ ).

(a) If $w$ has center $q$ on $A$, then the maximum length of a chain of valuations on $A[n]$ (resp., $L$ ) having center $q$ on $A$ and passing through $w$, is equal to

$$
\text { ht }\left(m(w) / q A_{w}\right)+\operatorname{tr} \operatorname{deg}_{A / q}^{k_{w}}
$$


(b) If $w$ extends $v$, then the maximum length of a chain of valuations on $A[n]$ (resp., $L$ ) extending $v$ is equal to

$$
\text { ht }\left(m(w) / m(v) A_{w}\right)+\operatorname{tr} \operatorname{deg}_{k_{v}}^{k_{w}} .
$$

Proposition 2.11. (a) Let $s$ be the maximal value of $\mathrm{ht}\left(m(w) / q A_{w}\right)+$ tr $\operatorname{deg}_{A / q}^{k_{w}}$, where $w$ runs through all valuations on $A[n]$ (resp., $K(n)$ ) with center $q$ on $A$. Let $t$ be the maximal value of $\operatorname{tr} \operatorname{deg}_{A / q}^{k_{v^{\prime}}}$, where $v^{\prime}$ runs through all valuations on $K$ with center $q$ on $A$. Then $s=n+t$.

(b) The value $n$ is the maximal value of $h t\left(m(w) / m(v) A_{w}\right)+\operatorname{trdeg}_{k_{v}}^{k_{w}}$, where $w$ runs through all valuations on $A[n]$ (resp., $K(n)$ ) extending $v$.

Proof. (a) Let $w$ be a valuation on $A[n]$ (resp., $K(n)$ ) with center $q$ on $A$ and let $\left(k_{1}, k_{2}\right) \in \mathbb{N}^{2}$ be such that $k_{1} \leq \operatorname{tr} \operatorname{deg}_{A / q}^{k_{w}}$ and $k_{2} \leq \operatorname{ht}\left(m(w) / q A_{w}\right)$. According to Theorems 2.4 and 2.6, there exists a chain of valuations $w=$ $w_{0}<\cdots<w_{k_{1}}$ on $A[n]$ (resp., $K(n)$ ) with center $q$ on $A$. By Corollary 2.8, there exists a chain of valuations $w_{k_{2}}<\cdots<w_{0}=w$ on $A[n](\operatorname{resp} ., K(n))$ with center $q$ on $A$. Thus, we have a chain of valuations $w_{k_{2}}<\cdots<w_{0}=w<$ $\cdots<w_{k_{1}}$ on $A[n]$ (resp., $K(n)$ ) with center $q$ on $A$, and

$$
k_{1}+k_{2} \leq \operatorname{trdeg} \operatorname{deg}_{A / q}^{k_{k_{2}}}=\operatorname{trdeg}_{k_{w_{k_{2}} \mid K}}^{k_{w_{k_{2}}}}+\operatorname{trdeg}_{A / q} k_{w_{k_{2}} \mid K} \leq \operatorname{trdeg}_{K}^{K(n)}+t \leq n+t,
$$

where $w_{k_{2} \mid K}$ is the restriction of $w_{k_{2}}$ to $K$. Consequently, $s \leq n+t$.

Conversely, take $k \leq t$. Then there exists a valuation $v^{\prime}$ on $K$ with center $q$ on $A$ such that $k \leq \operatorname{tr} \operatorname{deg}_{A / q}^{k_{v^{\prime}}}$. According to Proposition 2.1, there exists a valuation $w_{0}$ on $A[n]$ extending $v^{\prime}$ with $\operatorname{tr} \operatorname{deg}_{k_{v^{\prime}}}^{k_{w_{0}}}=n$, and

$$
n+k \leq \operatorname{tr} \operatorname{deg}_{k_{v^{\prime}}}^{k_{w_{0}}}+\operatorname{tr} \operatorname{deg}_{A / q}^{k} k_{v^{\prime}}=\operatorname{tr} \operatorname{deg}_{A / q}^{k w_{0}} \leq s
$$

(b) The proof follows immediately from (a) and the preceding results.

COROLLARY 2.12. If the transcendence degree of $L$ on $K$ is infinite, then there is no upper bound on $\operatorname{trdeg}_{A / q}^{k_{w}}+h t\left(m(w) / q A_{w}\right)$, with $w$ running through all valuations on $L$ with center $q$ on $A$.

PROoF. The proof is immediate from the preceding proposition.

3. The symbol $\delta((0), Q)$ in $A[n]$. Throughout this section, $A$ will be an integral domain, $(0) \neq q$ a prime ideal of $A, K$ the quotient field of $A$, and $n$ will be a nonnegative integer.

LEMMA 3.1. Let $Q$ be a superior of $q$ in $A[X]$ such that there exists $a \in A$ with $X-a \in Q$. Then, for each valuation $v$ of $K$ with center $q$ on $A$, there exists 
a valuation $w$ of $K(X)$ with center $Q$ on $A[X]$, extending $v$, and such that

$$
\operatorname{trdeg} \operatorname{der}_{\operatorname{Fr}(A[X] / Q)}^{k_{w}}=\operatorname{trdeg} \operatorname{Fr}(A / q)_{k_{v}}+1 .
$$

Proof. Let $\delta$ be a strictly positive element of the value group $G_{v}$ of $v$. We define the valuation $w$ on $K(X)$ as follows: if

$$
f(X)=b_{0}+\cdots+b_{n}(X-a)^{n},
$$

then

$$
w(f(X))=\inf \left\{v\left(a_{i}\right)+i \delta \mid i \in\{0, \ldots, n\}\right\}
$$

It is well known (see [1]) that $\operatorname{trdeg}_{k_{v}}^{k_{w}}=1$. We show that $w$ is a valuation on $A[X]$ with center $Q$ on $A[X]$. For $f(X)=b_{0}+\cdots+b_{n}(X-a)^{n} \in A[X]$, we have

$$
w(f(X))=\inf \left\{v\left(b_{i}\right)+i \delta \mid i \in\{0, \ldots, n\}\right\} \geq 0 .
$$

If $f(X) \in m(w) \cap A[X]$, then $b_{0} \in m(v) \cap A=q$ and $f(X) \in Q$.

Conversely, let $g(X)=a_{0}+\cdots+a_{m}(X-a)^{m} \in Q \subset A[X]$. For each $i \in$ $\{1, \ldots, m\}, v\left(a_{i}\right)+i \delta>0$ and $a_{0} \in m(v) \cap A=q$, hence

$$
w(g(X))=\inf \left\{v\left(a_{i}\right)+i \delta \mid i \in\{0, \ldots, m\}\right\}>0,
$$

that is, $g(X) \in m(w) \cap A[X]$. Thus, we have

$$
\operatorname{tr} \operatorname{deg}_{\operatorname{Fr}(A[X] / Q)}^{k_{w}}=\operatorname{tr} \operatorname{deg}_{\operatorname{Fr}(A / q)}^{k_{w}}=\operatorname{tr} \operatorname{deg}_{k_{v}}^{k_{w}}+\operatorname{tr} \operatorname{deg}_{\operatorname{Fr}(A / q)}^{k_{v}}=\operatorname{trdeg}_{\operatorname{Fr}(A / q)}^{k_{v}}+1 .
$$

LEMMA 3.2. Let $Q$ be a superior of $q$ in $A[X]$ and let $v$ be a valuation on $K$ with center $q$ on $A$. Then there exists a valuation $w$ on $K(X)$ with center $Q$ on $A[X]$ extending $v$ such that $\operatorname{tr} \operatorname{deg}_{\operatorname{Fr}(A[X] / Q)}^{k_{w}}=\operatorname{trdeg}_{\operatorname{Fr}(A / q)}^{k_{v}}+1$.

Proof. (a) Assume that $A$ is integrally closed in the algebraic closure $K^{\prime}$ of $K$. We have two different cases:

(1) $q$ is a maximal ideal of $A, Q^{\prime}=Q / q[X]$ is generated by $g(X)=X^{n}+$ $\bar{a}_{n-1} X^{n-1}+\cdots+\bar{a}_{0} \in(A / q)[X]$. Let $a_{i} \in A$ be a representant of $\bar{a}_{i} \in$ $(A / q)$. Then

$$
f(X)=a_{0}+\cdots+a_{n-1}(X-a)^{n-1}+X^{n}=\prod_{i=1}^{m}\left(X-r_{i}\right)^{\alpha_{i}} \in K[X] .
$$

Since $r_{i}$ is integral over $A$, so $r_{i} \in A$, and

$$
g(X)=\prod_{i=1}^{m}\left(X-\overline{r_{i}}\right)^{\alpha_{i}} \in Q^{\prime} .
$$


Then there exists $j \in\{1, \ldots, m\}$ such that $X-r_{j} \in Q$. We conclude by Lemma 3.2.

(2) Now, let $q$ be any prime ideal in $A$. Let $S=A-q$; we have $\left(S^{-1} Q\right)$ which is a superior to $q A_{q}$ in $A_{q}[X]$ and $A_{q}$ is integrally closed in $K^{\prime}$. The valuation $v$ has center $q A_{q}$ on $A_{q}$, so there exists a valuation $w$ of $K[X]$ with center $S^{-1} Q$ on $A_{q}[X]$ extending $v$, with

$$
\begin{aligned}
\operatorname{tr} \operatorname{deg}_{\operatorname{Fr}\left(A_{q}[X] / S^{-1} Q\right)}^{k_{w}} & =\operatorname{trdeg} \operatorname{deg}_{\operatorname{Fr}(A / q)}^{k_{v}}+1, \\
\operatorname{tr} \operatorname{deg}_{\operatorname{Fr}(A[X] / Q)}^{k_{w}} & =\operatorname{trdeg} \operatorname{deg}_{\operatorname{Fr}(A / q)}^{k_{v}}+1 .
\end{aligned}
$$

(b) Let $A^{\prime}$ be the integral closure of $A$ in the algebraic closure $K^{\prime}$ of $K$. Let $v^{\prime}$ be a valuation on $K^{\prime}$ extending $v$. The integral closure of $A$ in $K^{\prime}$ is the intersection of all the valuation rings on $K^{\prime}$ that contain $A$, as $v$ is a valuation on $A$, so $v^{\prime}$ is a valuation on $A^{\prime}$. Let $q^{\prime}$ be the center of $v^{\prime}$ on $A^{\prime}, q^{\prime} \cap A=q$, and $q^{\prime}[X] \cap A[X]=q[X]$. The closure $A^{\prime}[X]$ is integral over $A[X]$, so there exists a prime ideal $Q^{\prime}$ of $A^{\prime}[X]$ such that $Q^{\prime}$ is a superior of $q^{\prime}$, and $Q^{\prime}$ lies over $Q$. According to (a), there exists a valuation $w^{\prime}$ of $K^{\prime}(X)$ with center $Q^{\prime}$ on $A^{\prime}[X]$ extending $v^{\prime}$ with $\operatorname{tr} \operatorname{deg}_{\operatorname{Fr}\left(A^{\prime}[X] / Q^{\prime}\right)}^{k}=\operatorname{tr} \operatorname{deg}_{\operatorname{Fr}\left(A^{\prime} / q^{\prime}\right)}^{k_{v^{\prime}}}+1$. Let $w$ be the restriction of $w^{\prime}$ to $K(X) ; w$ is a valuation on $A[X]$,

$$
\begin{aligned}
m(w) \cap A[X] & =m\left(w^{\prime}\right) \cap K(X) \cap A[X] \\
& =m\left(w^{\prime}\right) \cap A^{\prime}[X] \cap A[X]=Q^{\prime} \cap A[X]=Q,
\end{aligned}
$$

and $w$ prolongs $v$. Also

$$
\operatorname{trdeg} \operatorname{deg}_{\operatorname{Fr}\left(A^{\prime} / q^{\prime}\right)}^{k_{v^{\prime}}}+\operatorname{tr} \operatorname{deg}_{\operatorname{Fr}(A / q)}^{\operatorname{Fr}\left(A^{\prime} / q^{\prime}\right)}=\operatorname{tr} \operatorname{deg}_{\operatorname{Fr}(A / q)}^{k_{v^{\prime}}}=\operatorname{tr} \operatorname{deg}_{k_{v}}^{k_{v^{\prime}}}+\operatorname{tr} \operatorname{deg}_{\operatorname{Fr}(A / q)}^{k_{v}} .
$$

It follows from Proposition 1.3 that $\operatorname{tr} \operatorname{deg}_{k_{v}}^{k_{v^{\prime}}} \leq \operatorname{tr} \operatorname{deg}_{K}^{K^{\prime}}=0$, hence

$$
\begin{aligned}
\operatorname{tr} \operatorname{deg}_{\operatorname{Fr}\left(A^{\prime} / q^{\prime}\right)}^{k_{v^{\prime}}} & =\operatorname{trdeg} \operatorname{deg}_{\operatorname{Fr}(A / q)}^{k_{v}}, \\
\operatorname{tr} \operatorname{deg}_{\operatorname{Fr}\left(A^{\prime}[X] / Q^{\prime}\right)}^{k_{w^{\prime}}^{\prime}} & =\operatorname{trdeg} \operatorname{deg}_{\operatorname{Fr}(A[X] / Q),}^{k_{w}}, \\
\operatorname{tr} \operatorname{deg}_{\operatorname{Fr}(A[X] / Q)}^{k_{w}} & =\operatorname{trdeg} \\
k_{\operatorname{Fr}(A / q)} & +1 .
\end{aligned}
$$

REMARK 3.3. Let $Q$ be a prime ideal of $A[X]$ lying over $q$. Then, for each valuation $v$ of $K$ with center $q$ on $A$, there exists a valuation $w$ of $K(X)$ extending $v$ and with center $Q$ on $A[X]$ such that

$$
\operatorname{tr} \operatorname{deg}_{\mathrm{Fr}(A[X] / Q)}^{k_{w}}=\operatorname{trdeg} \operatorname{deg}_{\mathrm{Fr}(A / q)}^{k_{v}}+\mathrm{ht}(Q / q[X]) .
$$

Indeed, Lemma 3.2 implies the case where $Q$ is a superior of $q$. If $Q=q[X]$, let $w$ be the canonical extension of $v$ to $K(X)$. It is well known (see Section 1 ) that $\operatorname{tr} \operatorname{deg}_{\operatorname{Fr}((A / q)[X])}^{k_{w}}=\operatorname{tr} \operatorname{deg}_{\operatorname{Fr}(A / q)}^{k_{v}}$. 
THEOREM 3.4. Let $Q$ be a prime ideal of $A[n]$ lying over $q$. Then, for each valuation $v$ of $K$ with center $q$ on $A$, there exists a valuation $w$ of $K(n)$ extending $v$ and with center $Q$ on $A[n]$ such that

$$
\operatorname{tr} \operatorname{deg}_{\operatorname{Fr}(A[n] / Q)}^{k_{w}}=\operatorname{tr} \operatorname{deg}_{\operatorname{Fr}(A / q)}^{k_{v}}+\text { ht }(Q / q[n]) .
$$

Proof. One proceeds by induction on $n$. The case $n=1$ follows from Remark 3.3. Assume that the statement is true for $n-1$. Let $Q_{1}=Q \cap A\left[X_{1}\right]$. Then there exists a valuation $w_{1}$ of $K\left(X_{1}\right)$ extending $v$, with center $Q_{1}$ on $A\left[X_{1}\right]$, and

$$
\operatorname{trdeg} \operatorname{deg}_{\operatorname{Fr}\left(A\left[X_{1}\right] / Q_{1}\right)}^{w_{1}}=\operatorname{trdeg} \operatorname{deg}_{\operatorname{Fr}(A / q)}^{k_{v}}+\operatorname{ht}\left(Q_{1} / q\left[X_{1}\right]\right),
$$

and there exists a valuation $w$ of $K\left(X_{1}\right)\left(X_{2}, \ldots, X_{n}\right)=K(n)$ extending $w_{1}$, with center $Q$ on $A[n]$, and

$$
\begin{aligned}
\operatorname{tr} \operatorname{deg}_{\operatorname{Fr}(A[n] / Q)}^{k_{w}} & =\operatorname{trdeg} \operatorname{deg}_{\operatorname{Fr}\left(A\left[X_{1}\right] / Q_{1}\right)}^{k_{w_{1}}}+\operatorname{ht}\left(Q / Q_{1}\left[X_{2}, \ldots, X_{n}\right]\right) \\
& =\operatorname{trdeg} \operatorname{der}_{\operatorname{Fr}(A / q)}^{k_{v}}+\operatorname{ht}\left(Q_{1} / q\left[X_{1}\right]\right)+\operatorname{ht}\left(Q / Q_{1}\left[X_{2}, \ldots, X_{n}\right]\right) .
\end{aligned}
$$

We conclude by remarking that

$$
\operatorname{ht}\left(Q_{1} / q\left[X_{1}\right]\right)+\operatorname{ht}\left(Q / Q_{1}\left[X_{2}, \ldots, X_{n}\right]\right)=\operatorname{ht}\left(Q / q\left[X_{1}, \ldots, X_{n}\right]\right) \text {. }
$$

NotAtion 3.5. Take $q_{1} \subset q_{2}$ in $\operatorname{Spec}(A)$. We will denote by $\delta\left(q_{1}, q_{2}\right)$ the maximal value $d$ for which there exists a valuation $v$ on $\operatorname{Fr}\left(A / q_{1}\right)$ with center $q_{2} / q_{1}$ on $A / q_{1}$ such that $\operatorname{tr} \operatorname{deg}_{\operatorname{Fr}\left(A / q_{2}\right)}^{k_{v}}=d$.

Jaffard has shown in [2] that $\delta\left((0), q_{2}\right)$ is the greatest number $n$ such that there exists a chain of valuations $v_{0}<\cdots<v_{n}$ on $A$ with center $q_{2}$ on $A$.

COROLlARY 3.6. Let $Q$ be a prime ideal of $A[n]$ lying over q. Then

$$
\delta((0), Q)=\delta((0), q)+\mathrm{ht}(Q / q[n])
$$

Proof. In the case where $Q=q[n]$, the result is well known (see [2]).

Suppose that $Q \neq q[n]$. For each valuation $v$ of $K$ with center $q$ on $A$, there exists a valuation $w$ of $K(n)$ with center $Q$ on $A[n]$, extending $v$, with

$$
\operatorname{trdeg} \operatorname{der}_{\operatorname{Fr}(A[n] / Q)}^{w_{w}}=\operatorname{tr} \operatorname{deg}_{\operatorname{Fr}(A / q)}^{k_{v}}+\operatorname{ht}(Q / q[n]) \leq \delta((0), Q),
$$

and consequently

$$
\delta((0), Q) \geq \delta((0), q)+\mathrm{ht}(Q / q[n])
$$


Conversely, let $w^{\prime}$ be a valuation on $K(n)$ with center $Q$ on $A[n]$ and let $v^{\prime}$ be its restriction to $K$. The valuation $v^{\prime}$ has center $q$ on $A$ and

$$
\operatorname{tr} \operatorname{deg}_{\operatorname{Fr}(A[n] / Q)}^{k_{w^{\prime}}}+\operatorname{tr} \operatorname{deg}_{\operatorname{Fr}(A / q)}^{\operatorname{Fr}(A[n] / Q)}=\operatorname{tr} \operatorname{deg}_{k_{v^{\prime}}}^{k_{w^{\prime}}}+\operatorname{tr} \operatorname{deg}_{\operatorname{Fr}(A / q)}^{k_{v^{\prime}}} \leq n+\delta((0), q),
$$

and therefore

$$
\begin{aligned}
\operatorname{tr} \operatorname{deg}_{\mathrm{Fr}(A[n] / Q)} & \leq \delta((0), q)+h t(Q / q[n]), \\
\delta((0), Q) & \leq \delta((0), q)+\operatorname{ht}(Q / q[n])
\end{aligned}
$$

Proposition 3.7. Let $q_{1}$ be a prime ideal of $A$ and $Q_{1} \subset Q_{2}$ two prime ideals of $A[n]$ lying over $q_{1}$. Then

$$
\delta\left(Q_{1}, Q_{2}\right)=\operatorname{ht}\left(Q_{2} / q_{1}[n]\right)-\operatorname{ht}\left(Q_{1} / q_{1}[n]\right)-1 .
$$

Proof. Let $T=A-q_{1}$. Then $T^{-1}\left(A[n] / Q_{1}\right)$ is an $\operatorname{Fr}\left(A / q_{1}\right)$-algebra of finite type, and therefore a Noetherian domain according to [3]. Then

$$
\begin{aligned}
\delta\left(Q_{1}, Q_{2}\right) & =\delta\left((0), Q_{2} / Q_{1}\right)=\delta\left((0), T^{-1}\left(Q_{2} / Q_{1}\right)\right) \\
& =\operatorname{ht}\left(T^{-1}\left(Q_{2} / Q_{1}\right)\right)-1=\operatorname{ht}\left(Q_{2} / Q_{1}\right)-1 \\
& =\operatorname{ht}\left(Q_{2} / q_{1}[n]\right)-h t\left(Q_{1} / q_{1}[n]\right)-1 .
\end{aligned}
$$

We finish this section studying the case of trivial valuations and we assume that $q=(0)$.

Proposition 3.8. Let $Q$ be a prime ideal of $A[n]$ lying over (0). Then there exists a valuation $w$ of $K(n)$ with center $Q$ on $A[n]$ such that

$$
\operatorname{tr} \operatorname{deg}_{\operatorname{Fr}(A[n] / Q)}^{k_{w}}= \begin{cases}\mathrm{ht}(Q)-1 & \text { if } Q \neq 0 \\ 0 & \text { if } Q=0\end{cases}
$$

Proof. If $Q=(0)$, then it suffices to take for $w$ the trivial valuation on $K(n)$. We suppose that $Q \neq(0)$. If $n=1$, then for each $w \in A(Q)$, according to the preceding result, $\operatorname{tr} \operatorname{deg}_{\operatorname{Fr}(A[X] / Q)}^{k_{w}} \leq \delta((0), Q)=0$, and therefore

$$
\operatorname{trdeg} \operatorname{deg}_{\mathrm{Fr}(A[X] / Q)}^{k_{w}}=\operatorname{ht}(Q)-1=0 .
$$

Take $n>1$, assume that the property holds for $n-1$, and let $Q_{1}=Q \cap$ $A\left[X_{1}\right]$. Then there exists a valuation $w_{1}$ of $K\left(X_{1}\right)$ with center $Q_{1}$ on $A\left[X_{1}\right]$ and $\operatorname{tr} \operatorname{deg}_{\operatorname{Fr}\left(A\left[X_{1}\right] / Q_{1}\right)}=0$. If $w_{1}$ is the trivial valuation, then there exists a valuation $w$ of $K\left(X_{1}\right)\left(X_{2}, \ldots, X_{n}\right)=K(n)$ with center $Q$ on $A\left[X_{1}\right]\left[X_{2}, \ldots, X_{n}\right]=A[n]$ and 
$\operatorname{tr} \operatorname{deg}_{\operatorname{Fr}(A[n] / Q)}^{k_{w}}=\operatorname{ht}(Q)-1$. If $w_{1}$ is not trivial, then $Q_{1} \neq(0)$ and it follows from Theorem 3.4 that there exists a valuation $w$ on $K(n)$ extending $w_{1}$ and with center $Q$ on $A[n]$, and

$$
\begin{aligned}
\operatorname{trdeg}_{\operatorname{Fr}(A[n] / Q)}^{k_{w}} & =\operatorname{trdeg} \operatorname{deg}_{\operatorname{Fr}\left(A\left[X_{1}\right] / Q_{1}\right)}^{k_{w_{1}}}+\operatorname{ht}\left(Q / Q_{1}\left[X_{2}, \ldots, X_{n}\right]\right) \\
& =\operatorname{ht}(Q)-\operatorname{ht}\left(Q_{1}\right) \\
& =\operatorname{ht}(Q)-1
\end{aligned}
$$

4. Valuations on $A[n]$ centered on the same ideal and extending the same valuation. Let $v$ be a valuation on $K$ with center $q$ on $A$ and $Q$ a prime ideal of $A[n]$ lying over $q$. We will use the following notation:

(a) $A(Q)=\{w \mid w$ is a valuation on $A[n]$ with center $Q\}$;

(b) $A(v)=\{w \mid w$ is a valuation on $A[n]$ extending $v\}$;

(c) $A(v, Q)=\{w \mid w$ is a valuation on $A[n]$ with center $Q$ extending $v\}$.

LEMMA 4.1. Let $w$ be a valuation on $K(n)$. Then $w$ is maximal in $A(v, Q)$ if and only if $w$ is maximal in $\left\{w^{\prime \prime} \mid w^{\prime \prime}\right.$ is a valuation on $A_{v}[n]$ with center $Q_{1}=$ $\left.m(w) \cap A_{v}[n]\right\}$ and $Q_{1}$ is maximal in $\left\{Q^{\prime} \mid Q^{\prime} \in \operatorname{Spec}\left(A_{v}[n]\right)\right.$ with $Q^{\prime} \cap A[n]=$ $Q\}$.

Proof. Suppose that $w$ is maximal in $A(v, Q)$. Assume that there exists $Q_{2} \in \operatorname{Spec}\left(A_{v}[n]\right)$ such that $Q_{1} \subset Q_{2}$ and $Q_{2} \cap A[n]=Q$. By Proposition 1.4, there exists a valuation $w_{2}$ on $A_{v}[n]$ with center $Q_{2}$, and $w<w_{2}$ such that $m\left(w_{2}\right) \cap A[n]=Q$ and $A_{v} \subseteq A_{w_{2}} \cap K \subseteq A_{w} \cap K=A_{v}$. Thus, $w_{2}$ extends $v$ with center $Q$ on $A[n]$, which is a contradiction.

Assume that $w<w^{\prime}$, with $w^{\prime}$ a valuation on $A_{v}[n]$ with center $Q_{1}$. Then $m\left(w^{\prime}\right) \cap A[n]=Q$ and $w^{\prime}$ extends $v$, that is, $w^{\prime}$ is a valuation on $A[n]$ with center $Q$ and extending $v$, which is impossible.

Conversely, if $w<w^{\prime}$ with $w^{\prime}$ a valuation on $A[n]$ extending $v$ and with center $Q$, then $m(w) \cap A_{v}[n]=Q_{1} \subset m\left(w^{\prime}\right) \cap A_{v}[n]$, but $\left(m\left(w^{\prime}\right) \cap A_{v}[n]\right) \cap$ $A[n]=m\left(w^{\prime}\right) \cap A[n]=Q$, which is again a contradiction.

REMARK 4.2. Take $w \in A(v, Q)$. Let $Q_{1}=m(w) \cap A_{v}[n]$ and assume that $Q_{1}$ is maximal in $\left\{Q^{\prime} \mid Q^{\prime} \in \operatorname{Spec}\left(A_{v}[n]\right), Q^{\prime} \cap A[n]=Q\right\}$. The ideal $Q_{1} /$ $m(v)[n]$ is maximal in $\left\{Q^{\prime} \mid Q^{\prime} \in \operatorname{Spec}\left(k_{v}[n]\right), Q^{\prime} \cap(A / q)[n]=Q / q[n]\right\}$. According to [5], we have

$$
\operatorname{ht}\left(Q_{1} / m(v)[n]\right)-\operatorname{ht}(Q / q[n])=\inf \left(\operatorname{tr} \operatorname{deg}_{A / q}^{k_{v}}, \operatorname{trdeg}_{A / q}^{A[n] / Q}\right) \text {. }
$$

THEOREM 4.3. For $w \in A(v, Q)$, the following assertions are equivalent:

(a) $w$ is maximal in $A(v, Q)$;

(b) $\inf \left(\operatorname{tr} \operatorname{deg}_{k_{v}}^{k_{w}}, \operatorname{tr} \operatorname{deg}_{A[n] / Q}^{k_{w}}\right)=0$.

Proof. First, suppose that $w$ is maximal in $A(v, Q)$. 
CASE 1. The transcendence degree of $k(v)$ on $k(q)$ is finite. Let $Q_{1}=m(w) \cap$ $A_{v}[n]$. Then

$$
\begin{aligned}
& \operatorname{tr} \operatorname{deg}_{A[n] / Q}^{k_{w}}+n-\operatorname{ht}(Q / q[n]) \\
& \quad=\operatorname{trdeg} \operatorname{deg}_{A / q}^{k_{w}}=\operatorname{tr} \operatorname{deg}_{A_{v}[n] / Q_{1}}^{k_{w}}+\operatorname{tr} \operatorname{deg}_{A[n] / Q}^{A_{v}[n] / Q_{1}}+n-\operatorname{ht}(Q / q[n])
\end{aligned}
$$

According to Lemma 4.1, $\operatorname{tr} \operatorname{deg}_{A_{v}[n] / Q_{1}}^{k_{w}}=0$, and

$$
\operatorname{tr} \operatorname{deg}_{A[n] / Q}^{A_{v}[n] / Q_{1}}+n-\operatorname{ht}(Q / q[n])=n-\operatorname{ht}\left(Q_{1} / m(v)[n]\right)+\operatorname{tr} \operatorname{deg}_{A / q}^{k_{v}},
$$

and therefore

$$
\begin{aligned}
\operatorname{tr} \operatorname{deg}_{A[n] / Q}^{A_{v}[n] / Q_{1}} & =\operatorname{ht}(Q / q[n])-\operatorname{ht}\left(Q_{1} / m(v)[n]\right)+\operatorname{trdeg}_{A / q}^{k_{v}}, \\
\operatorname{trdeg} & \operatorname{deg}_{A[n] / Q}^{k_{w}}= \\
& \operatorname{trdeg} \operatorname{de}_{A[n] / Q}^{A_{v}[n] / Q_{1}} \\
& \operatorname{ht}(Q / q[n])-\operatorname{ht}\left(Q_{1} / m(v)[n]\right)+\operatorname{trdeg}_{A / q}^{k_{v}} .
\end{aligned}
$$

Remark 4.2 implies that

$$
\operatorname{trdeg} \operatorname{deg}_{A[n] / Q}^{k_{w}}=\operatorname{trdeg}{ }_{A / q}^{k_{v}}-\inf \left(\operatorname{trdeg}_{A / q}^{k_{v}}, \operatorname{trdeg}_{A / q}^{A[n] / Q}\right) .
$$

If $\operatorname{tr} \operatorname{deg}_{A[n] / Q}^{k_{w}} \neq 0$, then $\operatorname{tr} \operatorname{deg}_{A[n] / Q}^{k_{w}}=\operatorname{tr} \operatorname{deg}_{A / q}^{k_{v}}+\operatorname{ht}(Q / q[n])-n$, and therefore

$$
\operatorname{tr} \operatorname{deg}_{A / q}^{k_{w}}=\operatorname{tr} \operatorname{deg}_{k_{v}}^{k_{w}}+\operatorname{tr} \operatorname{deg}_{A / q}^{k_{v}}=\operatorname{tr} \operatorname{deg}_{A / q}^{k_{v}},
$$

that is, $\operatorname{tr} \operatorname{deg}_{k_{v}}^{k_{w}}=0$.

CASE 2. The transcendence degree of $k(v)$ on $k(q)$ is infinite. We will show that $\operatorname{tr} \operatorname{deg}_{k_{v}}^{k_{w}}=0$. According to Lemma 4.1 and Remark 4.2,

$$
\operatorname{ht}\left(Q_{1} / m(v)[n]\right)-\operatorname{ht}(Q / q[n])=\operatorname{trdeg} A[n] / Q=n-\operatorname{ht}(Q / q[n])
$$

and therefore $\operatorname{ht}\left(Q_{1} / m(v)[n]\right)=n$. Thus,

$$
\operatorname{tr} \operatorname{deg}_{A_{v}[n] / Q_{1}}^{k_{w}}+\operatorname{tr} \operatorname{deg}_{A_{v} / m(v)}^{A_{v}[n] / Q_{1}}=\operatorname{tr} \operatorname{deg}_{k_{v}}^{k_{w}},
$$

so

$$
\operatorname{tr} \operatorname{deg}_{k_{v}}^{k_{w}}=\operatorname{tr} \operatorname{deg}_{A_{v} / m(v)}^{A_{v}[n] / Q_{1}}=n-h t\left(Q_{1} / m(v)[n]\right)=0 .
$$

Conversely, assume that $\inf \left(\operatorname{tr} \operatorname{deg}_{k_{v}}^{k_{w}}, \operatorname{tr} \operatorname{deg}_{A[n] / Q}^{k_{w}}\right)=0$. It follows from Corollary 2.9 that $w$ is maximal in $A(v, Q)$.

Proposition 4.4. If $w_{0} \in A(v, Q)$ is not maximal in $A(v, Q)$, then there exists $w_{1}$ in $A(v, Q)$ such that $w_{0}<w_{1}$ and $\operatorname{tr} \operatorname{deg}_{A[n] / Q}^{k_{w_{1}}}=\operatorname{trdeg}_{A[n] / Q}^{k_{w_{0}}}-1$. 
Proof. Let $Q_{0}=m\left(w_{0}\right) \cap A_{v}[n]$. According to Lemma 4.1, $\operatorname{trdeg}_{A_{v}[n] / Q_{0}}^{k_{w_{0}}} \neq$ 0 or $Q_{0}$ is not maximal in $\left\{Q^{\prime} \mid Q^{\prime} \in \operatorname{Spec}\left(A_{v}[n]\right)\right.$ with $\left.Q^{\prime} \cap A[n]=Q\right\}$.

If $\operatorname{trdeg}_{A_{v}[n] / Q_{0}}^{k_{w_{0}}} \neq 0$, then it follows from Lemma 2.3 that there exists a valuation $w_{1}$ on $A_{v}[n]$ with center $Q_{0}$ such that $w_{0}<w_{1}$ and $\operatorname{tr} \operatorname{deg}_{A_{v}[n] / Q_{0}}^{k_{w_{1}}}=$ $\operatorname{tr} \operatorname{deg}_{A_{v}[n] / Q_{0}}^{k_{w_{0}}}-1$, that is, $\operatorname{trdeg}_{A[n] / Q}^{k_{w_{1}}}=\operatorname{tr} \operatorname{deg}_{A[n] / Q}^{k_{w_{0}}}-1$. The valuation $w_{1}$ extends $v$ since $A_{v} \subseteq A_{w_{1}} \cap K \subseteq A_{w_{0}} \cap K=A_{v}$.

If $\operatorname{tr} \operatorname{deg}_{A_{v}[n] / Q_{0}}^{k_{w_{0}}}=0$, let $Q_{1}$ be a prime ideal of $A_{v}[n]$ lying over $Q$ with $Q_{0} \subset Q_{1}$ and $\operatorname{ht}\left(Q_{1} / Q_{0}\right)=1$. According to Proposition 1.4 and Theorem 2.4, there exists a valuation $w_{1}$ on $A_{v}[n]$ with center $Q_{1}$ such that $w_{0}<w_{1}$ and $\operatorname{tr} \operatorname{deg}_{A_{v}[n] / Q_{1}}^{k_{w_{1}}}=0 ; w_{1}$ is a valuation on $A[n]$ with center $Q$. Then

$$
\begin{aligned}
& \operatorname{trdeg}_{A[n] / Q}^{k_{w_{0}}}=\operatorname{trdeg} \operatorname{deg}_{A[n] / Q}^{A_{v}[n] / Q_{0}}=\operatorname{ht}(Q / q[n])-\operatorname{ht}\left(Q_{0} / m(v)[n]\right)+\operatorname{tr} \operatorname{deg}_{A / q}^{k_{v}} \text {, } \\
& \operatorname{tr} \operatorname{deg}_{A[n] / Q}^{k_{w_{1}}}=\operatorname{ht}(Q / q[n])-\operatorname{ht}\left(Q_{1} / m(v)[n]\right)+\operatorname{trdeg}_{A / q}^{k_{v}} \text {, }
\end{aligned}
$$

and therefore

$$
\begin{aligned}
& \operatorname{tr} \operatorname{deg}_{A[n] / Q}^{k_{w_{0}}}-\operatorname{trdeg}{ }_{A[n] / Q}^{k_{w_{1}}}=\operatorname{ht}\left(Q_{1} / m(v)[n]\right)-\operatorname{ht}\left(Q_{0} / m(v)[n]\right) \\
& =\mathrm{ht}\left(Q_{1} / Q_{0}\right)=1 \text {. }
\end{aligned}
$$

THEOREM 4.5. For $w \in A(v, Q)$, let $d(w, v, Q)=\operatorname{Sup}\{k \mid k \in \mathbb{N}, \exists k+1$ valuations $w=w_{0}<\cdots<w_{k}$ in $\left.A(v, Q)\right\}$. Then

$$
d(w, v, Q)=\inf \left(\operatorname{tr} \operatorname{deg}_{k_{v}}^{k_{w}}, \operatorname{tr} \operatorname{deg}_{A[n] / Q}^{k_{w}}\right) .
$$

Proof. Let $k=d(w, v, Q)$. According to Proposition 4.4, there exist $w_{0}<$ $\cdots<w_{k}$ in $A(v, Q)$, with $\operatorname{tr} \operatorname{deg}_{A[n] / Q}^{k_{w_{i}}}=\operatorname{trdeg}_{A[n] / Q} k_{w_{0}}-i$ for each $i \in\{0, \ldots, k\}$ and $w_{k}$ maximal in $A(v, Q)$, that is,

$$
\operatorname{trdeg}_{A[n] / Q}^{k_{w_{k}}}=\operatorname{trdeg}_{A / q}^{k_{v}}-\inf \left(\operatorname{tr} \operatorname{deg}_{A / q}^{k_{v}}, \operatorname{trdeg}_{A / q}^{A[n] / Q}\right)=\operatorname{trdeg}_{A[n] / Q}^{k_{w}}-k,
$$

and therefore

$$
\begin{aligned}
k & =\operatorname{tr} \operatorname{deg}_{A[n] / Q}^{k_{w}}-\operatorname{tr} \operatorname{deg}_{A / q}^{k_{v}}+\inf \left(\operatorname{tr} \operatorname{deg}_{A / q}^{k_{v}}, \operatorname{trdeg}_{A / q}^{A[n] / Q}\right) \\
& =\inf \left(\operatorname{tr} \operatorname{deg}_{k_{v}}^{k_{w}}, \operatorname{tr} \operatorname{deg}_{A[n] / Q}^{k_{w}}\right) .
\end{aligned}
$$

Proposition 4.6. For $w \in A(v, Q)$,

$$
\begin{aligned}
s & =\sup \left\{k \mid \exists k+1 \text { valuations } w_{k}<\cdots<w_{0}=w \text { on } A(v, Q)\right\} \\
& =h=\inf \left(\operatorname{ht}\left(m(w) / Q A_{w}\right)\right), \operatorname{ht}\left(m(w) / m(v) A_{w}\right) .
\end{aligned}
$$


Proof. Let $k \in \mathbb{N}$ and let $w_{k}<\cdots<w_{0}=w$ be valuations on $A(v, Q)$. As $A(v, Q) \subseteq A(v) \cap A(Q), k \leq \inf \left(\operatorname{ht}\left(m(w) / Q A_{w}\right), \operatorname{ht}\left(m(w) / m(v) A_{w}\right)\right)$, and consequently $s \leq h$.

The converse inequality is trivial if $s=\infty$. So let $s<\infty$ and assume $s<h$. Take $w_{k}<\cdots<w_{0}=w$ in $A(v, Q)$. Then

$$
\begin{aligned}
\operatorname{ht}\left(m(w) / Q A_{w}\right) & =\operatorname{ht}\left(m(w) / m\left(w_{k}\right)\right)+\mathrm{ht}\left(m\left(w_{k}\right) / Q A_{w_{k}}\right) \\
& =k+\mathrm{ht}\left(m\left(w_{k}\right) / Q A_{w_{k}}\right),
\end{aligned}
$$

and therefore $\mathrm{ht}\left(m\left(w_{k}\right) / Q A_{w_{k}}\right) \neq 0$, and there exists $w_{k+1}^{\prime} \in A(Q)$ with $w_{k+1}^{\prime}<$ $w_{k}$. As

$$
\begin{aligned}
\operatorname{ht}\left(m(w) / m(v) A_{w}\right) & =\operatorname{ht}\left(m(w) / m\left(w_{k}\right)\right)+\mathrm{ht}\left(m\left(w_{k}\right) / m(v) A_{w_{k}}\right) \\
& =k+\mathrm{ht}\left(m\left(w_{k}\right) / m(v) A_{w_{k}}\right),
\end{aligned}
$$

we have that ht $\left(m\left(w_{k}\right) / m(v) A_{w_{k}}\right) \neq 0$, and therefore there exists $w_{k+1}^{\prime \prime} \in A(v)$ with $w_{k+1}^{\prime \prime}<w_{k}$. Thus, $A_{w_{k}} \subset A_{w_{k+1}^{\prime}}, A_{w_{k}} \subset A_{w_{k+1}^{\prime \prime}}, A_{w_{k+1}^{\prime}}=\left(A_{w_{k}}\right)_{P^{\prime}}$, and $A_{w_{k+1}^{\prime \prime}}=$ $\left(A_{w_{k}}\right)_{P^{\prime \prime}}$ with $P^{\prime}$ and $P^{\prime \prime}$ two prime ideals of $A_{w_{k}}$. As $P^{\prime}$ and $P^{\prime \prime}$ are comparable, $A_{w_{k+1}^{\prime}} \subseteq A_{w_{k+1}^{\prime \prime}}$ or $A_{w_{k+1}^{\prime \prime}} \subseteq A_{w_{k+1}^{\prime}}$. If $w_{k+1}^{\prime \prime} \leq w_{k+1}^{\prime}, m\left(w_{k+1}^{\prime \prime}\right) \subseteq m\left(w_{k+1}^{\prime}\right)$ and $m(v)=m\left(w_{k+1}^{\prime \prime}\right) \cap A_{v} \subseteq m\left(w_{k+1}^{\prime}\right) \cap A_{v}$, that is, $m\left(w_{k+1}^{\prime}\right) \cap A_{v}=m(v)$ and $w_{k+1}^{\prime}$ extends $v$, it will be a contradiction since $w_{k+1}^{\prime} \in A(v, Q)$.

If $w_{k+1}^{\prime} \leq w_{k+1}^{\prime \prime}$, then $m\left(w_{k+1}^{\prime}\right) \subseteq m\left(w_{k+1}^{\prime \prime}\right) \subset m(w)$, and then $m\left(w_{k+1}^{\prime \prime}\right) \cap$ $A[n]=Q$ and $w_{k+1}^{\prime \prime} \in A(v, Q)$, which is again a contradiction.

We conclude that $s=h$ and finish the proof.

COROLLARY 4.7. For $w \in A(v, Q)$, the following assertions are equivalent:

(a) $w$ is minimal in $A(v, Q)$;

(b) $\inf \left(\mathrm{ht}\left(m(w) / Q A_{w}\right), \mathrm{ht}\left(m(w) / m(v) A_{w}\right)\right)=0$;

(c) $w$ is minimal in $A(Q)$ or in $A(v)$.

Notation 4.8. For each valuation $w$ on $A(v, Q)$, let $l(w)$ be the maximal length of a chain of valuations on $A(v, Q)$ passing through $w$. The maximum value of $l(w)$, where $w$ runs through the set $A(v, Q)$, will be denoted by $d(A(v, Q))$.

THEOREM 4.9. For each valuation $w$ on $A(v, Q)$,

(a) $l(w)=\inf \left(\operatorname{ht}\left(m(w) / Q A_{w}\right), \operatorname{ht}\left(m(w) / m(v) A_{w}\right)\right)+\inf \left(\operatorname{tr} \operatorname{deg}_{k_{v}}^{k_{w}}\right.$, $\left.\operatorname{tr} \operatorname{deg}_{A[n] / Q}^{k_{w}}\right)$;

(b) $d(A(v, Q))=\operatorname{tr} \operatorname{deg}_{k(q)}^{k v}+\mathrm{ht}(Q / q[n])$.

Proof. (a) The proof follows immediately from Theorem 4.5 and Proposition 4.6.

(b) Let $w^{\prime} \in A(v, Q)$. Then

$$
\operatorname{tr} \operatorname{deg}_{A[n] / Q}^{k_{w^{\prime}}}+n-\operatorname{ht}(Q / q[n])=\operatorname{tr} \operatorname{deg}_{k(q)}^{k_{w^{\prime}}}=\operatorname{tr} \operatorname{deg}_{k(v)}^{k_{w^{\prime}}}+\operatorname{tr} \operatorname{deg}_{k(q)}^{k_{v}},
$$


thus

$$
\begin{aligned}
\operatorname{trdeg}_{A[n] / Q}^{k_{w^{\prime}}} & =\operatorname{trdeg} \operatorname{deg}_{k(q)}^{k_{v}}+\operatorname{ht}(Q / q[n])+\left(\operatorname{trdeg}_{k(v)}^{k_{w^{\prime}}}-n\right) \\
& \leq \operatorname{trdeg} \operatorname{deg}_{k(q)}^{k_{v}}+\operatorname{ht}(Q / q[n]) .
\end{aligned}
$$

Therefore, $d(A(v, Q)) \leq \operatorname{tr} \operatorname{deg}_{k(q)}^{k v}+\mathrm{ht}(Q / q[n])$.

Theorem 3.4 implies the existence of $w \in A(v, Q)$ satisfying $\operatorname{tr} \operatorname{deg}_{A[n] / Q}^{k_{w}}=$ $\operatorname{tr} \operatorname{deg}_{k(q)}^{k_{v}}+h t(Q / q[n])$, and the converse inequality follows.

\section{REFERENCES}

[1] N. Bourbaki, Algèbre Commutative (chapitres 5 à 7), Masson, Paris, 1965 (French).

[2] P. Jaffard, Théorie de la Dimension dans les Anneaux de Polynomes, Mémor. Sci. Math., no. 146, Gauthier-Villars, Paris, 1960 (French).

[3] _ Valuations d'un anneau Noetherien et Théorie de la dimension, Algèbre et Théorie Nombres, vol. 13, Sem. P. Dubreil, M.-L. Dubreil-Jacotin et C. Pisot, 1961, p. 21 (French).

[4] S. Lang, Introduction to Algebraic Geometry, Interscience Publishers, New York, 1958.

[5] A. R. Wadsworth, The Krull dimensions of tensor products of commutative algebras over a field, J. London Math. Soc. (2) 19 (1979), no. 3, 391-401.

[6] O. Zariski and P. Samuel, Commutative Algebra. Vol. II, The University Series in Higher Mathematics, D. Van Nostrand, New York, 1960.

Rachid Chibloun: Département de Mathématiques et Informatique, Université Moulay Ismail, BP 4010, Béni M’hamed, Méknès, Morocco

E-mail address: chibloun@fsmek.ac .ma 


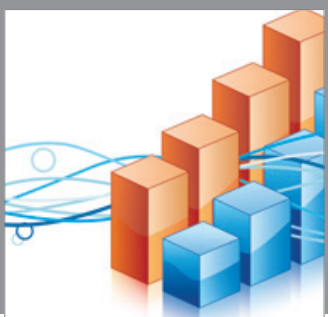

Advances in

Operations Research

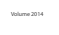

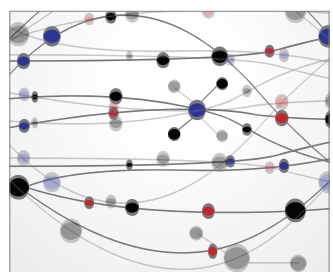

\section{The Scientific} World Journal
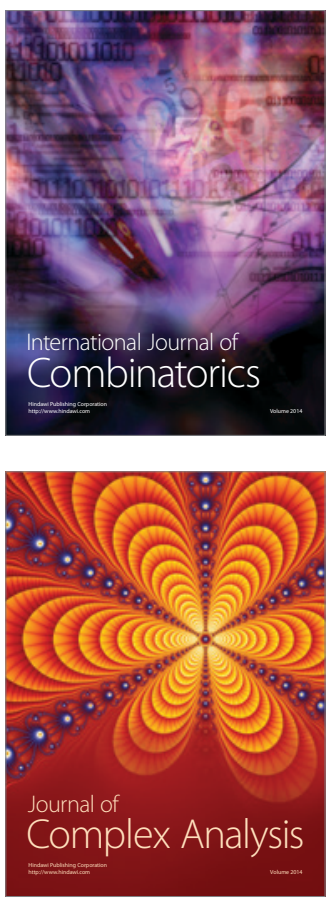

International Journal of

Mathematics and

Mathematical

Sciences
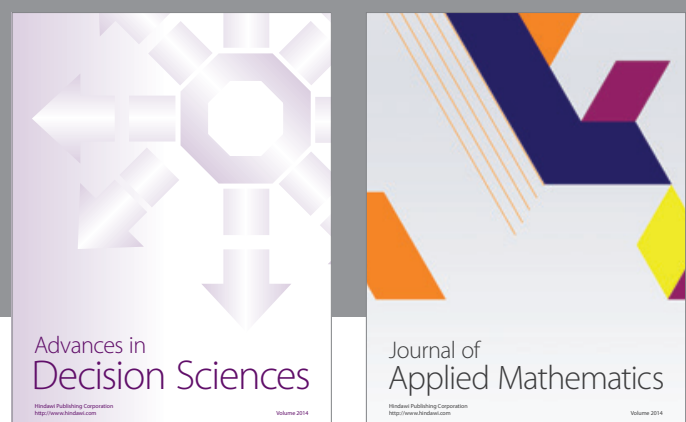

Journal of

Applied Mathematics
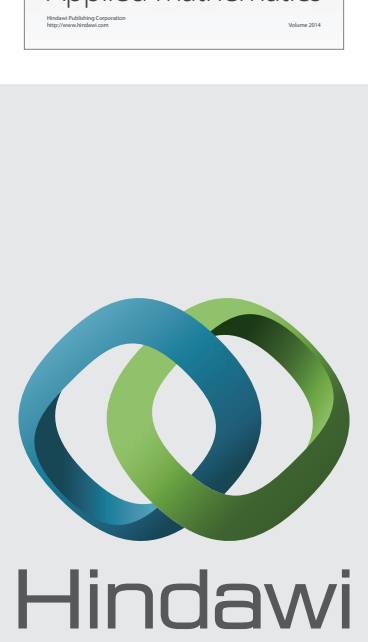

Submit your manuscripts at http://www.hindawi.com
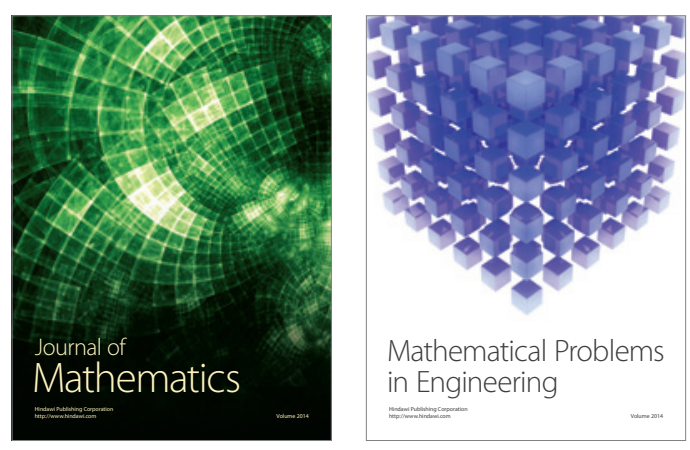

Mathematical Problems in Engineering
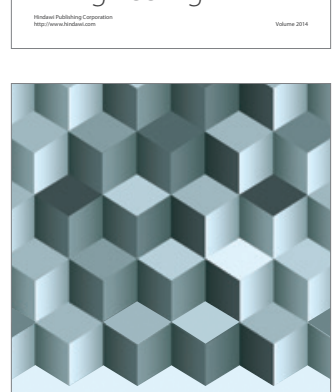

Journal of

Function Spaces
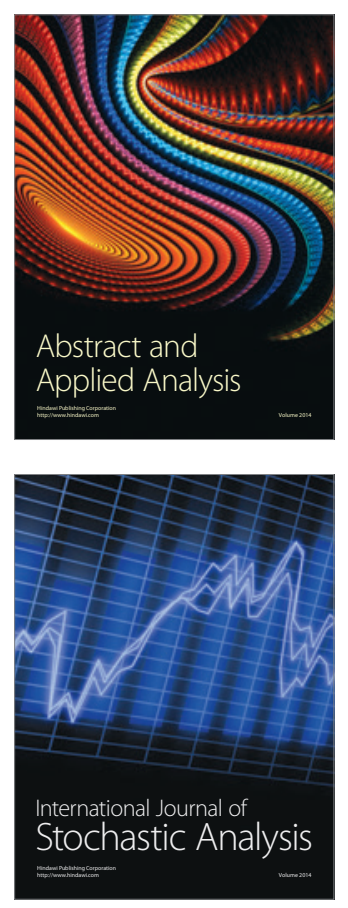

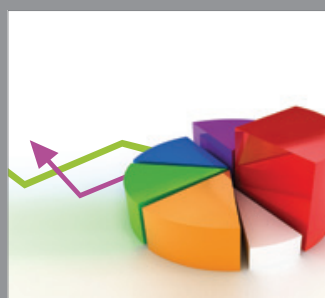

ournal of

Probability and Statistics

Promensencen
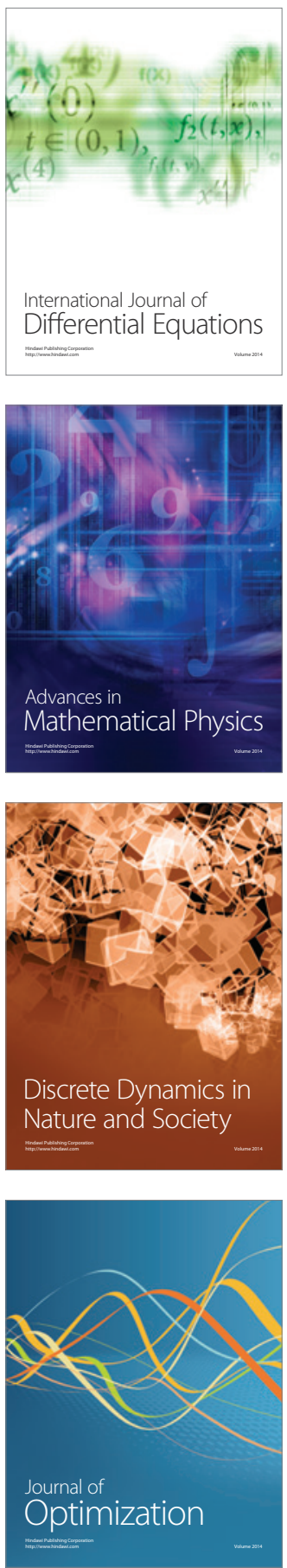